\title{
Inhibition of STAT3 signaling pathway by ursolic acid suppresses growth of hepatocellular carcinoma
}

\author{
TIANSHU LIU ${ }^{1}$, HAIYAN MA ${ }^{1}$, WEI SHI ${ }^{1}$, JIALIN DUAN $^{1}$, YINA WANG $^{1}$, CUNTAI ZHANG $^{2}$, \\ CHENGLONG LI ${ }^{3}$, JIAYUH LIN ${ }^{4}$, SHENG LI ${ }^{1}$, JIAGAO LV ${ }^{1}$ and LI LIN ${ }^{1}$ \\ ${ }^{1}$ Division of Cardiology, Department of Internal Medicine, ${ }^{2}$ Department of Geriatrics, Tongji Hospital, \\ Tongji Medical College, Huazhong University of Science and Technology, Wuhan, Hubei, P.R. China; \\ ${ }^{3}$ Department of Medicinal Chemistry, College of Pharmacy, University of Florida, Gainesville, FL; \\ ${ }^{4}$ Department of Biochemistry and Molecular Biology, University of Maryland School of Medicine, Baltimore, MD, USA
}

Received December 5, 2016; Accepted May 19, 2017

DOI: $10.3892 /$ ijo.2017.4035

\begin{abstract}
The signal transducer and activator of transcription 3 (STAT3) has been found to be constitutively active in liver cancer. There is no STAT3 inhibitors approved to be used clinically for the treatment or prevention of liver cancer. Some dietary compounds including ursolic acid (UA) have been reported to inhibit the growth of cancer cells. However, whether UA could inhibit STAT3 phosphorylation in hepatocellular carcinoma has not been reported. The inhibitory effects of UA on STAT3 phosphorylation, along with cell viability, migration, colony formation in vitro, as well as tumor growth in vivo were examined in human liver cancer cell lines. Our data showed that UA inhibited the P-STAT3 induced by interleukin-6 (IL-6) in Hep3B liver cancer cells which express very low basal level of P-STAT3. The constitutive STAT3 phosphorylation was also inhibited by UA in HEPG2, 7721 and Huh7 human liver cancer cell lines. UA decreased the expression of downstream target genes of STAT3, such as Bcl-2, Bcl-xl and survivin in general, with difference in these cell lines. UA also suppressed cell viability, cell migration and colony formation in liver cancer cells. Furthermore, UA suppressed STAT3 phosphorylation and HEPG2 tumor growth by oral daily treatment in vivo. UA, which exists widely in fruits and herbs, could inhibit STAT3 activation and the growth of human liver cancer cells in vitro and in vivo. It might be a potential health care product that could be used daily for
\end{abstract}

Correspondence to: Professor Li Lin or Professor Jiagao Lv, Division of Cardiology, Department of Internal Medicine, Tongji Hospital, Tongji Medical College, Huazhong University of Science and Technology, Wuhan Hubei 430030, P.R. China

E-mail: linli@tjh.tjmu.edu.cn; linlee271227@163.com

E-mail: lujiagao@tjh.tjmu.edu.cn

Abbreviations: STAT3, signal transducer and activator of transcription 3; IL-6, interleukin-6; UA, ursolic acid; JAK2, Janus kinases 2

Key words: ursolic acid, STAT3, IL-6, liver cancer, JAK2 prevention, as well as a promising candidate for chemotherapy of liver cancer.

\section{Introduction}

Hepatocellular carcinoma (HCC) is the fifth most common form of cancer and the most aggressive and frequently diagnosed malignancy of the liver. HCC is responsible for more than 600,000 deaths annually in the world. The main risk factors of HCC include hepatitis B virus infection, hepatitis $\mathrm{C}$ virus infection, alcohol abuse and tobacco. Recent data indicate that the mortality of primary liver cancer in China is increasing (1). Surgical resection has been considered the classical treatment, but only a small proportion of patients are diagnosed in time to have the chance for surgery. Therefore, there is a critical need for effective approaches for HCC treatment, especially in intermediate-stage and end-stage.

The signal transducer and activator of transcription (STAT) protein family have been shown to play an important role in tumor cell survival and proliferation (2). Among them, constitutive activation of STAT3 has been detected in a wide number of human cancer cell lines and primary tumors, including $50 \%$ of $\operatorname{HCC}(3,4)$. STAT3 can be activated by certain cytokines such as interleukin-6 (IL-6) (5), leukemia inhibitory factor (LIF) (6) and interferon- $\alpha$ (IFN- $\alpha$ ) (7). Among these inflammation factors inducing STAT3 phosphorylation, IL- 6 is regarded as one of the most vital cytokines in the studies published in the past few years. Patients with HCC show elevated levels of IL-6 in their serum compared with those with liver cirrhosis or healthy individuals (8). The homodimerization of the IL- 6 triggers a signaling cascade of phosphorylation of Janus kinases 2 (JAK2) and a downstream effector STAT3, followed by reciprocal dimerization of the Tyr705 phosphorylated STAT3 (9). Then, the STAT3 downstream target genes are activated, including Bcl-xl, Bcl-2 and survivin $(10,11)$. It has been shown that aberrant activation of IL-6/JAK2/STAT3 signaling pathway plays an important role in pathogenesis and progress of liver cancer (12). However, there is still no JAK2/STAT3 inhibitors approved to be used clinically for the treatment or prevention of HCC. 
Ursolic acid (UA) is natural triterpenoid compound found in plants, herbs and other kinds of food. It has been identified to play noteworthy role in anti-inflammatory, hepatoprotective and antiallergic activities. It suppresses cell proliferation of various types of cancer, including multiple myeloma (13), colon (14), breast (15), pancreatic (16) and prostate cancer (17) by inhibiting the STAT3 signaling pathway. It has not been reported previously that UA inhibits phosphorylation of JAK2 and STAT3 in liver cancer cell lines and suppresses growth of hepatocellular carcinoma. Aberrant activation of IL-6/JAK2/ STAT3 signaling pathway has been shown to play a role in pathogenesis of liver cancer, which features high malignancy and relative high resistance to chemotherapy. Due to the fact that different types of cancer may exhibit diverse resistance or sensitivity to antitumor agents, it is urgent to detect the levels of STAT3 phosphorylation and the effect of UA in liver cancer cells despite previous studies describing the effect of UA in other cancers.

\section{Materials and methods}

Human liver cancer cell lines. Human liver cancer cell lines (Hep3B, HEPG2, SSMC-7721 and Huh7) were purchased from the American Type Culture Collection (ATCC; Manassas, VA, USA) and cultured in Dulbecco's modified Eagle's medium (DMEM) high glucose supplemented with $10 \%$ fetal bovine serum (FBS; Hyclone Laboratories, Inc., Logan, UT, USA) and $1 \%$ penicillin/streptomycin. All cancer cell lines were cultured in a humidified $37^{\circ} \mathrm{C}$ incubator with $95 \%$ air and $5 \% \mathrm{CO}_{2}$.

Compounds. Ursolic acid (UA) was purchased from SigmaAldrich (St. Louis, MO, USA). It was dissolved in sterile dimethyl sulfoxide (DMSO) to get $20 \mathrm{mM}$ stock solution for cell experiments, and stored at $-20^{\circ} \mathrm{C}$ until use. IL-6, IFN- $\alpha$ and LIF were from Cell Signaling Technology (Danvers, MA, USA).

Cell viability assay. Cell viability was measured using the MTT assay. The MTT cell viability assay kits was from Promoter Biotechnology Ltd. (Wuhan, China) UA was added in cultured medium to form different concentration $(0,10,25$, $30,40$ and $50 \mu \mathrm{M})$. Four liver cancer cells types $(4,500 /$ well in 96-well plates) were incubated with the medium at $37^{\circ} \mathrm{C}$ for $24 \mathrm{~h}$ with UA. Then, $10 \mu \mathrm{l}$ MTT solution was added to each well and liver cancer cells were cultured at $37^{\circ} \mathrm{C}$. After $4 \mathrm{~h}$, $100 \mu 1$ MTT formanzan solution was added to each well and a further 4-h incubation followed. The optical density (OD) was measured at a wavelength of $570 \mathrm{~nm}$. The treated cells viability was calculated as follows: (OD of UA group-OD of blank group/ OD of DMSO group-OD of blank group) x $100 \%$.

Moreover, MTT was performed to determine if the effect of UA on cell migration was due to its ability to inhibit cell viability. Huh7 cells were treated with UA for $4 \mathrm{~h}$, then medium with UA was removed and replaced with fresh medium for additional $36 \mathrm{~h}$ of incubation without UA. Then, $10 \mu \mathrm{l}$ MTT solution was added to each well and Huh7 cells were cultured at $37^{\circ} \mathrm{C}$. After $4 \mathrm{~h}, 100 \mu \mathrm{l}$ MTT formanzan solution was added to each well and a further 4-h incubation followed. The optical density (OD) was measured at a wavelength of $570 \mathrm{~nm}$. The treated cells viability was calculated as follows: (OD of UA
group-OD of blank group/OD of DMSO group-OD of blank group) $\mathrm{x} 100 \%$.

Colony formation. Liver cancer cells were counted and plated in 6-well cell culture plates and pretreated with different concentrations of UA for $4 \mathrm{~h}$ at $37^{\circ} \mathrm{C}$. The cells were then washed with phosphate-buffered saline (PBS) twice and seeded on $10-\mathrm{cm}$ plates for 14 days in culture. After that, cells were fixed with cold methanol for $15 \mathrm{~min}$ and stained with $0.5 \%$ crystal violet ( $25 \%$ methanol) at room temperature for $10 \mathrm{~min}$. Finally, the plates were washed with distilled water and dried.

Wound healing assay. Liver cell lines were plated in 6-well plate (with three lines at the external bottom of each well) and cultured in DMEM)/high glucose supplemented with $10 \%$ FBS (HyClone Laboratories) and 1\% penicillin/streptomycin. When the cells grew to a confluence of $100 \%$, the monolayer cells were scratched using a $10-\mu 1$ pipette tip and rinsed with PBS to remove floating cells. Then the cells were treated with varying concentrations of UA ( 25 and $50 \mu \mathrm{M})$ or DMSO for $4 \mathrm{~h}$. Images of wound closure were taken at the crossing point of lines and scratches by an inverted microscope until the wound treated with DMSO was completely closed.

Transwell assay. Cells $\left(5 \times 10^{4}\right)$ pretreatment of UA for $4 \mathrm{~h}$ were suspended in $200 \mu 1$ serum-free DMEM medium and seeded into the upper chamber of each insert. Then, $600 \mu \mathrm{l}$ of DMEM containing $10 \%$ FBS was added to a 24 -well plate. After incubation at $37^{\circ} \mathrm{C}$ for $24 \mathrm{~h}$, the cells that migrated were fixed and stained for $30 \mathrm{~min}$ in a dye solution containing $0.1 \%$ crystal violet and $20 \%$ methanol.

Western blot analysis. Cancer cells were treated with UA (10, 25 and $50 \mu \mathrm{M}$ ) or DMSO for $12 \mathrm{~h}$. After the treatments, cells were collected. Cancer cells were serum-starved in media without FBS for $12 \mathrm{~h}$ before being treated with UA $(10,25$, or $50 \mu \mathrm{M})$ or DMSO for $4 \mathrm{~h}$ and incubated with IL-6 $(50 \mathrm{ng} / \mathrm{ml})$, LIF $(25 \mathrm{ng} / \mathrm{ml})$ or IFN- $\alpha(25 \mathrm{ng} / \mathrm{ml})$ for $30 \mathrm{~min}$. Then the cells were collected and washed with cold PBS and lysed on ice in a modified RIPA buffer (1\% Triton X-100, $1 \%$ deoxycholate, $0.1 \%$ SDS) containing protease inhibitors ( $1 \mathrm{mM}$ PMSF), subjected to SDS-PAGE. Proteins were transferred onto PVDF membrane and probed with antibodies (Cell Signaling Technology). Membranes were probed with a 1:1,000 dilution of primary antibodies (Cell Signaling Technology) against phospho-specific STAT3 (Tyrosine 705, \#9131), phosphoindependent STAT3 (\#4904), phospho-specific STAT1 (Tyr 701, \#8217), phospho-specific STAT2 (Tyr 690, \#4441), phospho-independent STAT2 (\#4597), phospho-specific JAK2 (Tyr 1007/1008,\#3776), phospho-specific Akt (Ser473,\#9271), phospho-independent Akt (\#9272), phospho-specific Erk1/2 (Thr 202/Tyr 204, \#9101), cleaved caspase-3 (Asp175, \#9661), Bcl-2 (\#2876), survivin (\#2803), Bcl-xl (\#2762) and GAPDH (\#2118). Phospho-independent STAT1 (\#D120084) was purchased from Sangon Biotech, Co., Ltd. (Shanghai, China). HRP-conjugated secondary antibodies were from Promotor Biotechnology Ltd. The specific proteins were detected using an enhanced chemiluminescence (ECL) western blotting kit according to the manufacturer's instructions. 
A

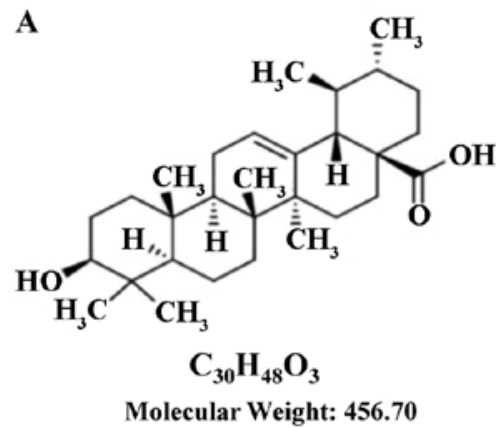

B

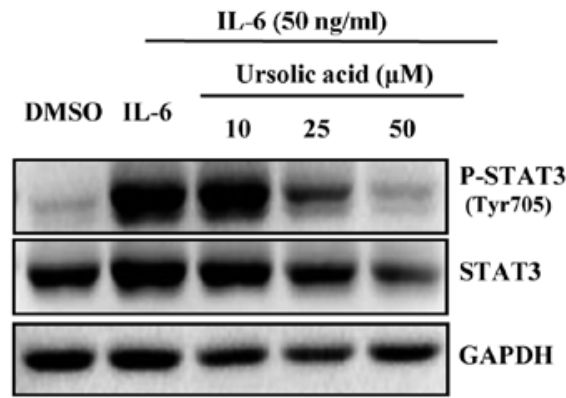

Hep3B

Figure 1. (A) The structure of ursolic acid (UA). (B) UA inhibited STAT3 phosphorylation induced by IL-6 in Hep3B liver cancer cell line. With pre-treatment of UA for $4 \mathrm{~h}$ and IL-6 (50 $\mathrm{ng} / \mathrm{ml})$ induced for another $30 \mathrm{~min}$, STAT3 phosphorylation was inhibited in a dose-dependent manner.

Immunofluorescence staining. Cells were seeded on sterile glass slides and grew for $24 \mathrm{~h}$. Hep3B cells were pretreated with UA for $4 \mathrm{~h}$ after serum-free overnight, then IL-6 was added for another $30 \mathrm{~min}$. After the treatments, the cells were washed with ice-cold PBS buffer, and were fixed with ice-cold methanol at room temperature for $15 \mathrm{~min}$. After three washings with ice-cold PBS buffer, the cells were permeabilized and blocked with PBS buffer containing 0.1\% Triton X-100 and $0.5 \%$ normal goat serum at room temperature for at least $1 \mathrm{~h}$. Then the cells were probed with rabbit antibody to phosphorylated STAT3 (1:50 dilution) at $4^{\circ} \mathrm{C}$ overnight. After the overnight incubation, the cells were washed with PBS buffer containing $0.1 \%$ Tween-20. The cells were incubated with Cy3-conjugated anti-rabbit secondary antibody (1:100; Jackson ImmunoResearch Laboratories, Inc., West Grove, PA, USA) at room temperature for $1 \mathrm{~h}$. Cells were incubated for $5 \mathrm{~min}$ at room temperature with DAPI (Vector Laboratories, Burlingame, CA, USA) to stain nuclei and observed using an inverted fluorescence microscope.

Mouse xenograft tumor model. All animal studies were conducted in accordance with the standard procedures approved by the Institutional Animal Care and Use Committee of Tongji Hospital, Huazhong University of Science and Technology. Known as a classic liver cancer xenograft model, human liver cancer cell line, HEPG2 $\left(10^{7}\right.$ cells in $100 \mu \mathrm{l}$ of sterile PBS and Matrigel), were injected subcutaneously into the right flank region of mice (4-6 weeks of age, 18-22 g). After tumor development, the mice were then randomized into two groups: i) DMSO as vehicle control; and ii) $60 \mathrm{mg} / \mathrm{kg} \mathrm{UA}$ ( $1.2 \mathrm{mg}$ of UA powder dissolved in a $100 \mu \mathrm{l}$ of mixture of $10 \mu \mathrm{l}$ DMSO and $90 \mu \mathrm{l}$ sterile water, $\sim 2.6 \mu \mathrm{mol} \mathrm{UA}$ in $100 \mu \mathrm{l}$ ). Each mouse was given daily $\sim 100 \mu \mathrm{l}$ mixture. Vehicle and UA groups were orally treated once daily for consecutive 15 days. Tumor growth was determined by measuring the length (L) and width $(\mathrm{W})$ of the tumor every other day with a caliper. In addition, tumor volume was calculated on the basis of the following formula: volume $=(\pi / 6) \times \mathrm{L} \mathrm{x} \mathrm{W}^{2}$. The mice were sacrificed after 15 days of treatment. On the 15 th day, the body weight of mice was measured. The tumors were harvested after mice were euthanized. We took images of the tumors and measured the weight of tumors. Then tumors were stored at $-80^{\circ} \mathrm{C}$. Tumor tissue homogenates were lysed and separated by
SDS-PAGE. The expression of STAT3, its downstream target genes Bcl-2 and cleaved caspase-3 in xenograft tumors was examined by western blot assay. A portion of tumor tissues were fixed by using formalin and embedded in paraffin. The expression of P-STAT3 (Tyr 705) and Bcl-2 was also examined by immunohistochemistry (IHC) staining. TUNEL (terminal deoxynucleotidyl transferase dUTP nick end labeling) assay was used to detect apoptosis in xenograft tumors.

Statistical analysis. The data are presented as the mean \pm SD for at least three independent experiments. Statistical analysis was performed with SPSS software (version 13.0). The significant differences between any of the two groups were evaluated by one-way analysis of ANOVA. Statistical significance was defined as $\mathrm{P}<0.05$.

\section{Results}

Ursolic acid inhibits STAT3 phosphorylation induced by IL-6 in Hep3B liver cancer cells. The structure of UA is shown in Fig. 1A. IL-6 is a growth factor and induces STAT3 phosphorylation (18). UA inhibited STAT3 phosphorylation induced by IL-6 $(50 \mathrm{ng} / \mathrm{ml})$ in a dose-dependent manner in Hep3B cells. There was marked inhibition of phosphorylation of STAT3 with UA $(50 \mu \mathrm{M})$ for $4 \mathrm{~h}$, but less significantly on STAT3 (Fig. 1B).

Ursolic acid does not inhibit the phosphorylation of STAT3 induced by LIF, but inhibits the phosphorylation of STAT1, STAT2 and STAT3 induced by IFN- $\alpha$ in Hep3B liver cancer cells. Members of STATs family including STAT1 to STAT6 are found in many kinds of cells and tissues as important mediators of cytokine signaling. LIF is expressed in HCC but less than IL-6, activating STAT3 pathway in liver cancer cells (19). As a member of the type I IFN family IFN- $\alpha$ affects intracellular signaling through the Jak/Stat pathway. In HCC, IFN- $\alpha$ can induce activation of STAT1 (20), STAT2 (21) and STAT3 (7). Our results showed that UA did not inhibit the phosphorylation of STAT3 and total STAT3 induced by LIF (Fig. 2A). But it could inhibit the STAT1, STAT2 and STAT3 phosphorylation induced by IFN- $\alpha$ in Hep3B liver cancer cell line and had a less significant effect on STAT1, STAT2 and STAT3 (Fig. 2B). 
A

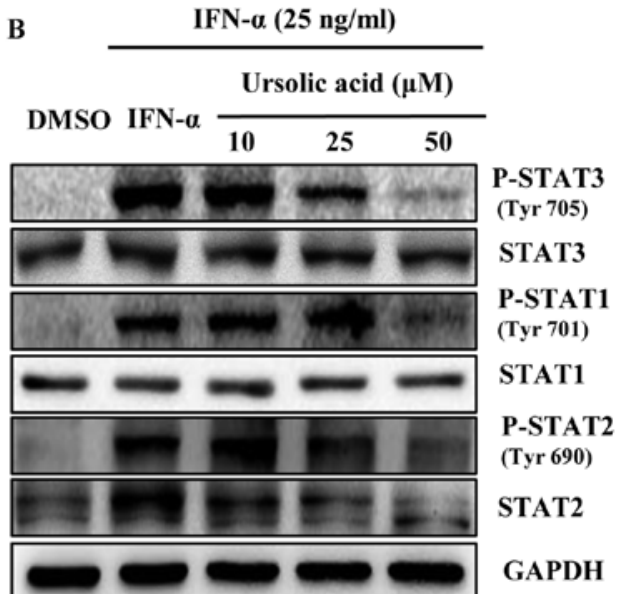

Hep3B

Figure 2. Ursolic acid (UA) does not affect the phosphorylation of STAT3 induced by LIF, but inhibits phosphorylation of STAT3 induced by IFN- $\alpha$, also inhibits other STAT phosphorylation, including STAT1 and STAT2 induced by IFN- $\alpha$. (A) UA could not inhibit the phosphorylation of STAT3 at Tyr705 induced by LIF ( $25 \mathrm{ng} / \mathrm{ml})$, (B) but inhibited the phosphorylation of STAT1 at Tyr701, STAT2 at Tyr690 and STAT3 at Tyr705 induced by IFN- $\alpha$ ( $25 \mathrm{ng} / \mathrm{ml})$.

A

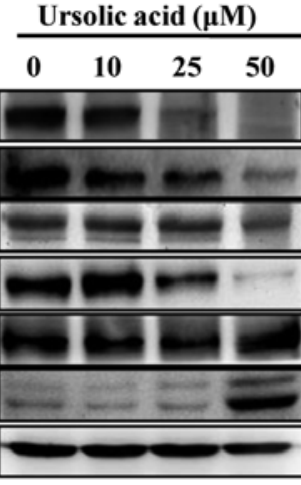

HEPG2

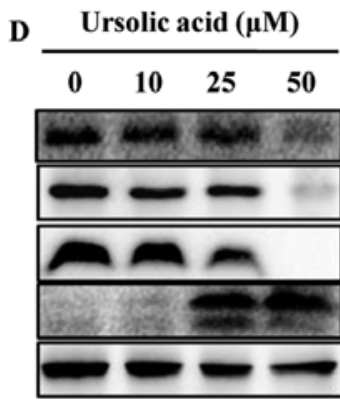

HEPG2
B

P-JAK2
(Tyr1007/1008)
P-STAT3
(Tyr705)
STAT3
P-Akt
(Ser47)
Akt
P-Erk
(Thr 202/ Tyr 204)
GAPDH

GAPDH

Bcl-xl
Bcl-2
Survivin
Cleaved
caspase 3
GAPDH

$\mathbf{E}$

Ursolic acid $(\mu \mathrm{M})$

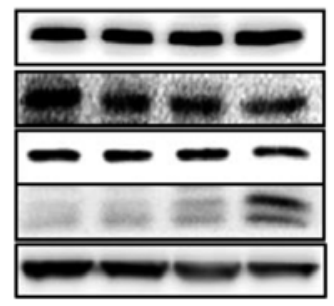

7721

P-JAK2
(Tyr1007/1008)
P-STAT3
(Tyr705)
STAT3
P-Akt
(Ser473)
Akt
P-Erk
(Thr 202/ Tyr 204)
GAPDH

GAPDH

7721

Bcl-xl

Bcl-2

Survivin

Cleaved

caspase 3

GAPDH

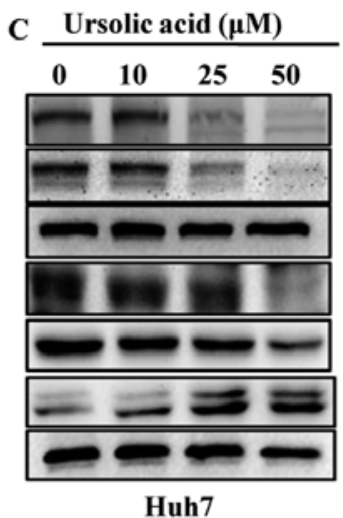

P-JAK2
(Tyr1007/1008)
P-STAT3
(Tyr705)
STAT3
P-Akt
(Ser47)
Akt
P-Erk
(Thr 202/ Tyr 204)
GAPDH

Huh7

\begin{tabular}{cccc} 
F & \multicolumn{4}{c}{ Ursolic acid $(\mu M)$} \\
\cline { 2 - 5 } & $\mathbf{1 0}$ & 25 & 50
\end{tabular}

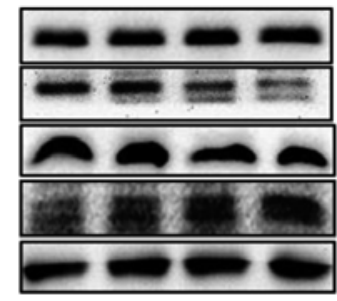

Bcl-xl

Bcl-2

Survivin

Cleaved

caspase 5

GAPDH

Huh7

Figure 3. Ursolic acid (UA) inhibits constitutive phosphorylation of JAK2 and STAT3 in liver cancer cell lines. With the treatment of UA for $12 \mathrm{~h}$, phosphorylation of JAK2 and STAT3 were tested by western blot analysis. (A-C) The phosphorylation of JAK2 and STAT3 was inhibited by UA in HEPG2, 7721 and Huh7 liver cancer cells. UA inhibited Akt phosphorylation and induced Erk phosphorylation in liver cancer cell lines. Under the same conditions, Akt phosphorylation was completely inhibited by UA at $50 \mu \mathrm{M}$. But P-Erk was induced in a concentration-dependent manner in HEPG2, 7721 and Huh7 cells. (D-F) Cleavage of caspase-3 was increased by UA. The level of survivin, Bcl-2 and Bcl-xl after treatment of UA was different in HEPG2, 7721 and Huh7 liver cancer cell lines. Bcl-2 expression was reduced in the Huh7 and HEPG2 cell line while Bcl-xl and survivin expression were markedly reduced in the HEPG2 cells. In 7721 cells, neither Bcl-xl, nor Bcl-2 or survivin showed reduced expression. Based on the results, it was possible that different liver cancer cells responded differently to UA.

Ursolic acid inhibits JAK2 and STAT3 phosphorylation in HEPG2, 7721 and Huh7 cancer cells. UA inhibited constitutive STAT3 phosphorylation in HEPG2, 7721 and Huh7 liver cancer cell lines in a dose-dependent manner but less significantly on the levels of total STAT3 (Fig. 3A-C). STAT3 has been reported to be activated by JAK2 (9). In our results, UA inhibited phosphorylation of JAK2 in a dose-dependent manner in HEPG2, 7721 and Huh7 liver cancer cells. The level of survivin, Bcl-2 and Bcl-xl after treatment of UA was different in HEPG2, 7721 and Huh7 liver cancer cells. Bcl-2 


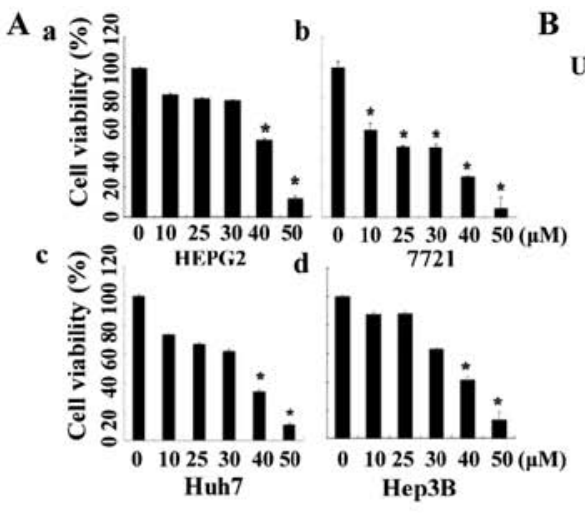

D Ursolic acid $(\mu \mathrm{M})$

0

25

50

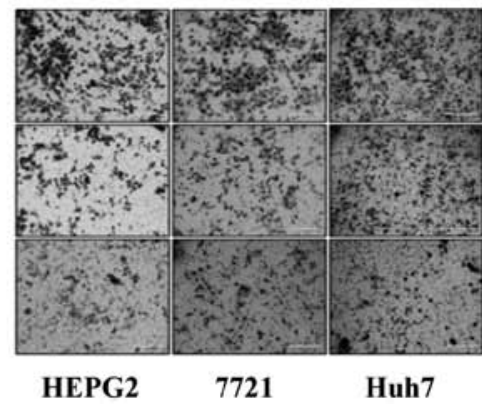

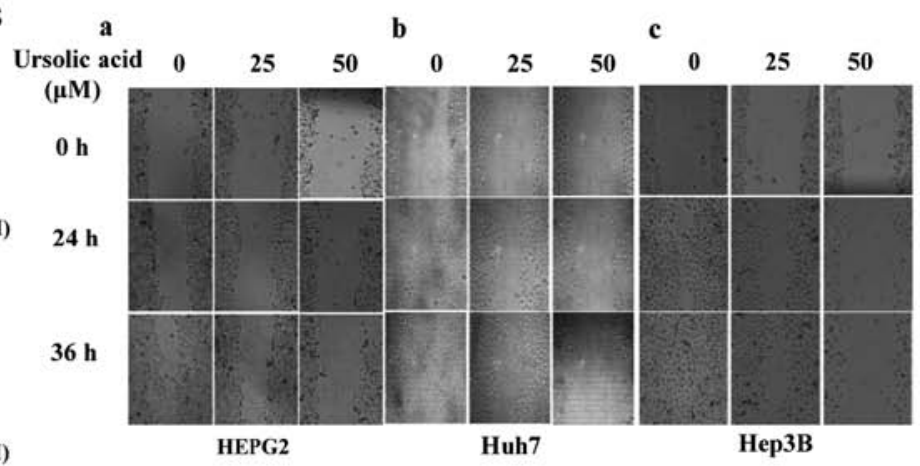

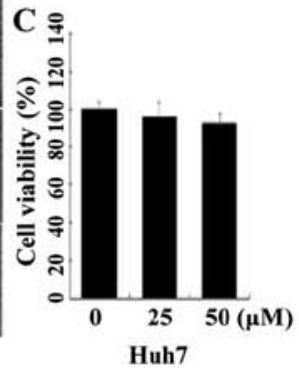

E Ursolic acid

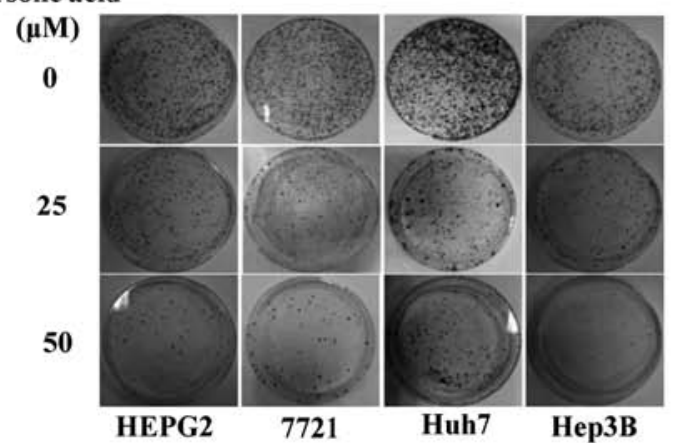

Figure 4. Ursolic acid (UA) inhibits the viability of liver cancer cells. (A) MTT assay showed that the viability of liver cancer cells was inhibited with the treatment of UA for $24 \mathrm{~h}$ in HEPG2 (A-a), 7721 (A-b), Huh7 (A-c) and Hep3B (A-d) liver cancer cells ("P<0.05). (B) UA inhibited wound-healing assay in HEPG2 (B-a), Huh7 (B-b) and Hep3B (B-c) cancer cells. (C) MTT assay showed that UA did not significantly inhibit the cell viability of Huh7 at the same dosages and time-points used in the migration assay. Thus, the ability of UA to inhibit cell migration may not be due to its ability to inhibit cell viability. (D) Transwell assay was applied to determine effect of UA on cell invasive ability in HEPG2, 7721 and Huh7 cell lines. It showed that UA (50 $\mu \mathrm{M})$ could significantly inhibit cell invasion in HEPG2, 7721 and Huh7 liver cancer cell lines. Cell colony formation was investigated after treatment with UA for $4 \mathrm{~h}$. (E) UA suppressed the colony formation of HEPG2, 7721, Huh7 and Hep3B liver cancer cell lines.

expression was reduced in the Huh7 and HEPG2 cells while $\mathrm{Bcl}-\mathrm{xl}$ and survivin expression were only markedly reduced in the HEPG2 cell line. In 7721 cells, neither Bcl-xl, nor Bcl-2 or survivin showed reduced expression (Fig. 3D-F). Based on the results, it was possible that different liver cancer cells responded differently to UA. We also detected other signaling pathways such as Akt and Erk. As shown in Fig. 3A-C, we demonstrated that UA inhibited the phosphorylation of Akt at Ser473 and less significantly on Akt. UA also increased expression levels of cleaved caspase-3 (Fig. 3D-F). The levels of P-Erk were increased after UA treatment in a dose-dependent manner in liver cancer cells as shown in Fig. 3A-C.

Ursolic acid inhibits cell viability. Our data showed that UA also inhibited the cell viability of four liver cancer cell lines (Fig. 4A). Cell viability of HEPG2 (A-a), 7721 (A-b) and Huh7 (A-c) liver cancer cells was decreased by $50 \mu \mathrm{M}$ of UA treatment, with a similar effect on Hep3B (A-d) cells $(\mathrm{P}<0.05)$.

Ursolic acid inhibits cell migration and invasion in liver cancer cells. We next evaluated the effect of UA on cell migration by wound healing assay in liver cancer cells. As shown in Fig. 4B, UA suppressed cell migration in a dose-dependent manner in HEPG2 (B-a), Huh7 (B-b) and Hep3B (B-c). MTT assay was carried out to determine if the effect of UA on cell migration was due to its ability to inhibit cell viability. The time-points (4 h with UA and incubation for additional $36 \mathrm{~h}$ without UA) were also used in the wound healing assay. The ability of UA to inhibit cell migration did not seem to be due to an inhibition of cell viability (Fig. 4C). Transwell assay was performed to determine the effect of UA on cell invasion in HEPG2, 7721 and Huh7 cancer cells. It showed that UA $(50 \mu \mathrm{M})$ could significantly inhibit cell invasion in cell lines mentioned here (Fig. 4D).

UA inhibits the colony formation in liver cancer cells. We also investigated the effect of UA on cell colony formation. The results showed that UA markedly inhibited the colony formation in HEPG2, 7721, Huh7 and Hep3B liver cancer cell lines (Fig. 4E).

UA inhibits STAT3 activation in the nucleus in Hep3B liver cancer cells. In Hep3B cells treated with IL-6, STAT3 was activated in the nucleus. Pre-treated with UA at $25 \mu \mathrm{M}$ for $4 \mathrm{~h}$, cells were then induced by IL-6 $(50 \mathrm{ng} / \mathrm{ml})$ for another 30 min and P-STAT3 was detected by immunofluorescence staining. These results indicated that P-STAT3 induced by IL-6 in nucleus could be inhibited by UA in Hep3B cells (Fig. 5).

Ursolic acid suppresses tumor growth in HEPG2 mouse xenograft model in vivo. HEPG2 xenograft experiments 


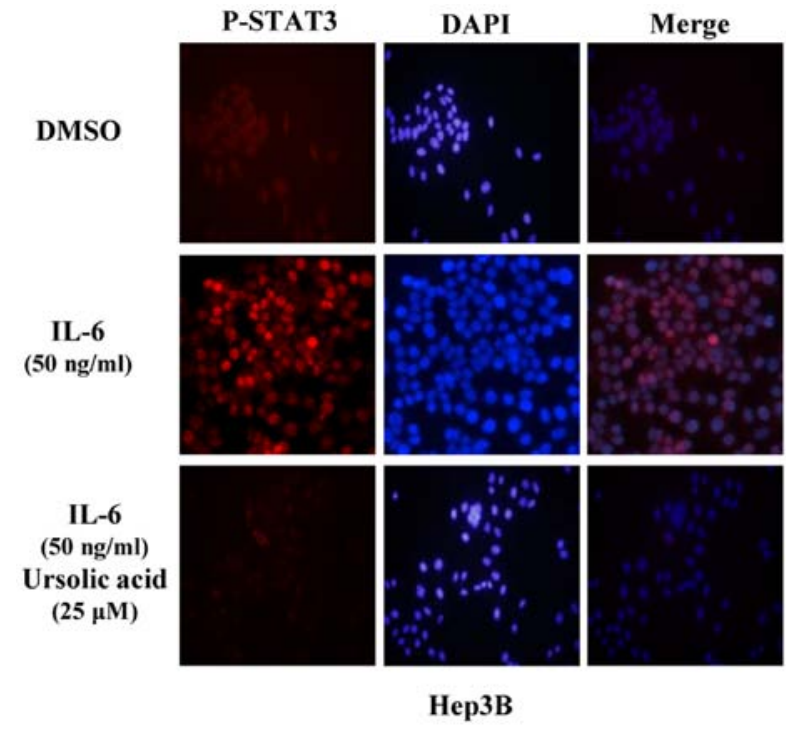

Figure 5. Ursolic acid (UA) inhibits STAT3 activation in nucleus induced by IL-6 in Hep3B cancer cells. In Hep3B cells treated with IL-6, STAT3 was activated in the nucleus. Pre-treated with UA at $25 \mu \mathrm{M}$ for $4 \mathrm{~h}$, cells were induced by IL- $6(50 \mathrm{ng} / \mathrm{ml})$ for another $30 \mathrm{~min}$ and P-STAT3 was detected by immunofluorescence staining. P-STAT3 induced by IL-6 in the nucleus was inhibited by UA in Hep3B cells.

were performed to explore whether UA could inhibit tumor growth in vivo (22). As shown in Fig. 6A, the tumor volume of mice given daily $60 \mathrm{mg} / \mathrm{kg}$ UA was decreased compared with vehicle group, measured by caliper every other day. After mice were euthanized, we isolated tumor tissue as shown in Fig. 6B. The tumor weight of UA group was significantly lower than that of vehicle group, accompanied by decreased percent of tumor/body weight (Fig. 6C; $\mathrm{P}<0.05$ ). Over the course of treatment, body weight was shown in Fig. 6D. UA inhibited STAT3 phosphorylation, decreased Bcl-2 and induced cleavage of caspase-3 in xenografts (Fig. 6E). In addition, UA also inhibited STAT3 phosphorylation, decreased the expression of Bcl-2 as shown by IHC staining and induced apoptosis by using TUNEL assay in xenograft tumors (Fig. 6F). These results suggested that UA inhibited P-STAT3 and suppressed tumor growth in mice, indicating that UA might be a potent compound in suppressing tumor growth in vivo.

\section{Discussion}

There are studies on the inhibition of STAT3 phosphorylation (both constitutive and IL-6 induced) by UA in myeloma (13), colon cancer (14) and prostate cancer cells (16). As is known, liver cancer features high malignancy and rapid progress, in addition to its relatively high resistance to chemotherapy. It has been reported that elevated level of IL-6 was expressed in serum of patients with HCC compared with healthy individuals (8), and different types of cancer exhibit different resistance and sensitivity to antitumor agents. However, it has not been reported previously that UA inhibits phosphorylation of JAK2 and STAT3 in liver cancer cell lines and suppresses growth of hepatocellular carcinoma, in which aberrant activation of IL-6/JAK2/STAT3 signaling pathway plays an important role in pathogenesis and progress. In the present study, we demonstrated that UA decreased JAK2 and STAT3 phosphorylation in liver cancer cell lines, and inhibited cell viability, cell migration and colony formation. Moreover, UA inhibited tumor growth in HEPG2 liver cancer xenograft mice. Thus, UA may have potential as therapeutic agent for liver cancer. In addition, our results showed that UA could inhibit STAT3 phosphorylation induced by IL-6, and also inhibit P-STAT1, 2, 3 induced by IFN- $\alpha$ in Hep3B. However, UA did not affect P-STAT3 induced by LIF. It suggested that the inhibitory effects of UA on P-STAT3 induced by diverse stimuli were different, which were possibly linked to different binding targets of IL-6, LIF and IFN- $\alpha$. Leukemia inhibitory factor (LIF) is a pleiotropic factor belonging to the IL-6 superfamily of cytokines. It is also expressed in HCC but less than IL-6. LIF can activate STAT3 pathway in liver cancer cells (19). Interferon- $\alpha$ (IFN- $\alpha$ ) is a member of the type I IFN family known for their antiviral activity (23). In HCC, the activation of STAT1 pathway via IFN- $\alpha$ might have inhibitory effect of hepatocellular carcinoma (20). IFN- $\alpha$ can also induce oncogenic signaling of STAT3 (7) and STAT2 (21). Although IL-6, LIF and IFN- $\alpha$ induce STAT3 activation, their receptor and binding targets are totally different. It has been reported that IL- 6 induces homodimerization of gp130 by 32 F6 (5), LIF leads to heterodimerization of gp130 with the LIF receptor (LIFR) by B-R3 (6). The IFN- $\alpha$ receptor consists of two subunits, IFN- $\alpha \mathrm{R} 1$ and IFN- $\alpha \mathrm{R} 2$, which form a heterodimer upon IFN- $\alpha$ stimulation (20). The effect of UA on P-STAT3 induced by LIF or IFN- $\alpha$ has not been reported before. Our data suggested that UA had different effects on STAT3 activation induced by different cytokines, indicating that UA might bind different residues of the different receptors of IL-6, LIF and IFN- $\alpha$. These might be helpful to find the exact mechanism or molecular target by which UA inhibits STAT3 phosphorylation in our future studies. UA might be used as a leading compound to develop new IL-6/gp130 inhibitors with higher selectivity and potency. As mentioned above, UA might also inhibit the activation of other STATs in vitro, which might have opposite effects on tumor growth. However, our in vivo experiments revealed that UA suppressed growth in a xenograft model as shown in Fig. 6.

Furthermore, we also detected other signaling pathways such as Erk and Akt. The Erk signaling pathway can be activated in response to a diverse range of extracellular stimuli including mitogens and cytokines (24). Akt plays a critical role in cell survival and apoptosis of liver cancer (25). Our results showed that UA inhibited the phosphorylation of Akt at Ser473. The levels of P-Erk were increased after treatment of UA in a dose-dependent manner in liver cancer cells. UA is a natural compound and may play a role in various signaling pathways including Akt and Erk besides STAT3. In this study, UA inhibited P-JAK2 and P-STAT3 at a concentration as low as $25 \mu \mathrm{M}$, but inhibited P-Akt at $50 \mu \mathrm{M}$. Thus, our results showed that UA inhibited P-Akt, but inhibited P-STAT3 more markedly, while increased P-Erk. Our data suggested that inhibition of STAT3 phosphorylation is one of the mechanism that participated in inhibitory effect of UA in liver cancer cell lines.

In our previous study, we examined the effect of UA on HCT116 and SW480 (human colon cancer cells). UA could inhibit P-STAT3 activation and cell viability significantly at $25 \mu \mathrm{M}$ in HCT116 and SW480 cells (14). In human multiple 
A
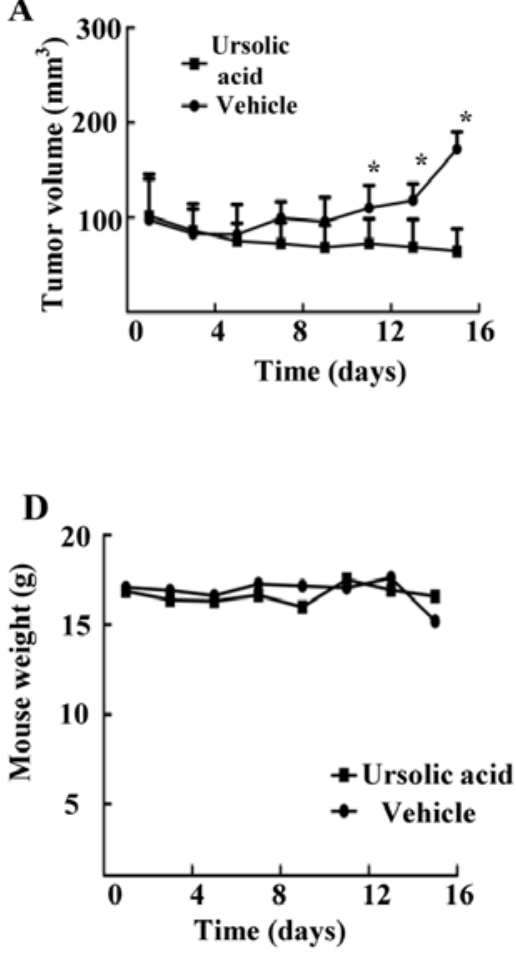

B

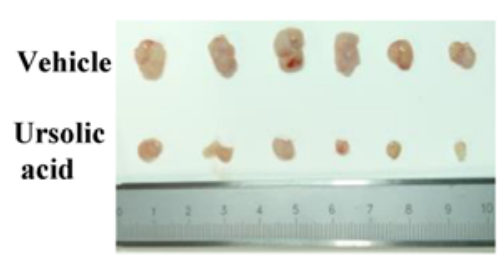

E Vehicle Ursolic acid

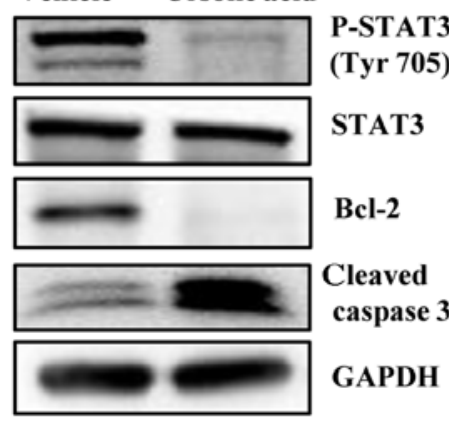

$\mathrm{C}^{\mathrm{a}}$

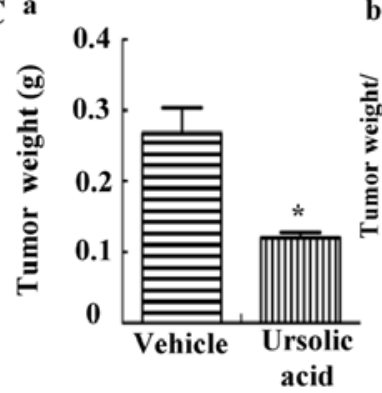

b

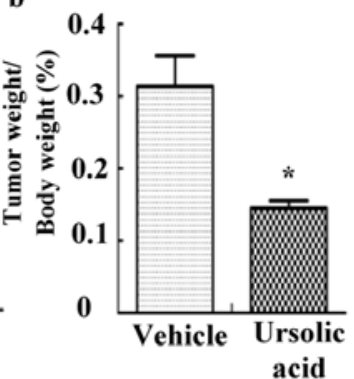

Figure 6. Ursolic acid (UA) inhibits tumor growth of HEPG2 liver cancer cells in vivo. (A) UA decreased tumor volume compared with vehicle group. (B) After mice were euthanized, we isolated tumor tissue. (C) The tumor weight and the percent of tumor/body weight of UA group were significant lower than that of vehicle group. (D) Body weights over the course of treatment. (E) UA inhibited P-STAT3 and Bcl-2, but not total STAT3, and induced caspase-3 cleavage in xenograft tumor model in vivo. (F) UA also inhibited STAT3 phosphorylation, decreased the expression of Bcl-2 as shown by IHC staining and induced apoptosis by TUNEL. ( $\mathrm{P}<0.05)$.

myeloma, UA inhibited P-STAT3 activation in U266 cells and cell viability in U266, MM1.S and RPMI 8826 at $25 \mu \mathrm{M}$ (13). In human pancreatic cancer, UA inhibited cell growth and proliferation at $20 \mu \mathrm{M}$ in AsPC-1, MIA PaCa-2 and Panc-28 (16). Our results showed that STAT3 phosphorylation could be inhibited at $25 \mu \mathrm{M}$ which was higher than other reported cancer cells, suggesting liver cancer cells might be more malignant and more severely resistant to UA than other cancer cells. It is of interest to note that the level of survivin, Bcl-2 and Bcl-xl after treatment of UA was different in HEPG2, 7721 and Huh7 liver cancer cells. Bcl-2 expression was reduced in the Huh7 and HEPG2 cell line while Bcl-xl and survivin expression were only markedly reduced in the HEPG2 cell line. In 7721 cells, neither Bcl-xl, nor Bcl-2 or survivin showed reduced expression. Based on the results, it was possible that different liver cancer cell lines responded differently to UA. In this study, we did not design experiments to explore the different effect of UA on different liver cancer cell lines, but it does have the potential to become a novel point in our future studies.

Ursolic acid is a pentacyclic triterpenoid compound in the form of free acid, which widely exists in apple peels, herb medicines and many other edible plants. Our data showed that UA inhibited constitutively activated STAT3 and Akt in HCC cell lines. We also found that UA suppressed tumor growth in HEPG2 liver cancer xenograft mice. Thus, UA has the potential to be used clinically as a candidate for liver cancer therapy with relatively low toxicity and strong biological stability compared to other artificial designed compounds. In addition, UA might be used to prevent the initiation of HCC. Because it is extracted from natural plants and fruits, such as apples, loquat and olive, people might take UA in their daily lives to reduce the risk of HCC. Also in our results, the mice treated with DMSO were not as strong as UA treated group, which suggested the protecting effect of UA. In conclusion, pure UA might be developed as a health care product to prevent liver cancer in future.

In summary, we found that UA, a natural compound from some plants and fruits, could suppress STAT3 phosphorylation, cell viability in vitro and xenograft tumor growth in vivo in HCC. The safety of UA, as natural food ingredient, can be preserved to be advantage for clinical treatment. UA is a plantderived agent and can be modified to design new inhibitors as antitumor compounds with better cellular permeability or bioavailability and less side-effects. UA as well as its analogues might be developed to meet the current FDA standards for future clinical testing.

\section{Acknowledgements}

The present study was supported by the National Natural Science Foundation of China to L.L. (No. 81372402, 81001005 and 81570416) and S.L. (No. 81570337), the Outstanding Young Investigator Foundation of Tongji Hospital (YXQN009) and the Fundamental Research Fund for the Central Universities, HUST (No. 0118540019) to L.L. 


\section{References}

1. Wang R, Chen XZ, Zhang MG, Tang L and Wu H: Incidence and mortality of liver cancer in mainland China: Changes in first decade of 21st century. Hepatogastroenterology 62: 118-121, 2015.

2. Bowman T, Garcia R, Turkson J and Jove R: STATs in oncogenesis. Oncogene 19: 2474-2488, 2000.

3. Buettner R, Mora LB and Jove R: Activated STAT signaling in human tumors provides novel molecular targets for therapeutic intervention. Clin Cancer Res 8: 945-954, 2002.

4. Subramaniam A, Shanmugam MK, Perumal E, Li F, Nachiyappan A, Dai X, Swamy SN, Ahn KS, Kumar AP, Tan BK, et al: Potential role of signal transducer and activator of transcription (STAT)3 signaling pathway in inflammation, survival, proliferation and invasion of hepatocellular carcinoma. Biochim Biophys Acta 1835: 46-60, 2013.

5. Chevalier S, Fourcin M, Robledo O, Wijdenes J, PouplardBarthelaix A and Gascan H: Interleukin-6 family of cytokines induced activation of different functional sites expressed by gp130 transducing protein. J Biol Chem 271: 14764-14772, 1996.

6. Hermanns HM, Radtke S, Haan C, Schmitz-Van de Leur H, Tavernier J, Heinrich PC and Behrmann I: Contributions of leukemia inhibitory factor receptor and oncostatin $\mathrm{M}$ receptor to signal transduction in heterodimeric complexes with glycoprotein 130. J Immunol 163: 6651-6658, 1999.

7. Wang L, Jia D, Duan F, Sun Z, Liu X, Zhou L, Sun L, Ren S, Ruan Y and Gu J: Combined anti-tumor effects of IFN- $\alpha$ and sorafenib on hepatocellular carcinoma in vitro and in vivo. Biochem Biophys Res Commun 422: 687-692, 2012.

8. Ataseven H, Bahcecioglu IH, Kuzu N, Yalniz M, Celebi S, Erensoy A and Ustundag B: The levels of ghrelin, leptin, TNF-alpha, and IL-6 in liver cirrhosis and hepatocellular carcinoma due to HBV and HDV infection. Mediators Inflamm 2006: 78380, 2006.

9. Song B, Zhan H, Bian Q and Gu J: Piperlongumine inhibits gastric cancer cells via suppression of the JAK1,2/STAT3 signaling pathway. Mol Med Rep 13: 4475-4480, 2016.

10. Wei LH, Kuo ML, Chen CA, Chou CH, Lai KB, Lee CN and Hsieh CY: Interleukin-6 promotes cervical tumor growth by VEGF-dependent angiogenesis via a STAT3 pathway. Oncogene 22: 1517-1527, 2003.

11. Lin L, Hutzen B, Li PK, Ball S, Zuo M, DeAngelis S, Foust E, Sobo M, Friedman L, Bhasin D, et al: A novel small molecule, LLL12, inhibits STAT3 phosphorylation and activities and exhibits potent growth-suppressive activity in human cancer cells. Neoplasia 12: 39-50, 2010.

12. Mohan CD, Bharathkumar H, Bulusu KC, Pandey V, Rangappa S, Fuchs JE, Shanmugam MK, Dai X, Li F, Deivasigamani A, et al: Development of a novel azaspirane that targets the Janus kinasesignal transducer and activator of transcription (STAT) pathway in hepatocellular carcinoma in vitro and in vivo. $\mathrm{J}$ Biol Chem 289: 34296-34307, 2014.
13. Pathak AK, Bhutani M, Nair AS, Ahn KS, Chakraborty A, Kadara H, Guha S, Sethi G and Aggarwal BB: Ursolic acid inhibits STAT3 activation pathway leading to suppression of proliferation and chemosensitization of human multiple myeloma cells. Mol Cancer Res 5: 943-955, 2007.

14. Wang W, Zhao C, Jou D, Lü J, Zhang C, Lin L and Lin J: Ursolic acid inhibits the growth of colon cancer-initiating cells by targeting STAT3. Anticancer Res 33: 4279-4284, 2013.

15. Heo TH, Wahler J and Suh N: Potential therapeutic implications of IL-6/IL-6R/gp130-targeting agents in breast cancer. Oncotarget 7: 15460-15473, 2016.

16. Prasad S, Yadav VR, Sung B, Gupta SC, Tyagi AK and Aggarwal BB: Ursolic acid inhibits the growth of human pancreatic cancer and enhances the antitumor potential of gemcitabine in an orthotopic mouse model through suppression of the inflammatory microenvironment. Oncotarget 7: 13182-13196, 2016.

17. Shanmugam MK, Rajendran P, Li F, Nema T, Vali S, Abbasi T, Kapoor S, Sharma A, Kumar AP, Ho PC, et al: Ursolic acid inhibits multiple cell survival pathways leading to suppression of growth of prostate cancer xenograft in nude mice. J Mol Med (Berl) 89: 713-727, 2011.

18. Kawano M, Hirano T, Matsuda T, Taga T, Horii Y, Iwato K, Asaoku H, Tang B, Tanabe $\mathrm{O}$, Tanaka $\mathrm{H}$, et al: Autocrine generation and requirement of BSF-2/IL- 6 for human multiple myelomas. Nature 332: 83-85, 1988.

19. Zhang JF, He ML, Fu WM, Wang H, Chen LZ, Zhu X, Chen Y, Xie D, Lai P, Chen G, et al: Primate-specific microRNA-637 inhibits tumorigenesis in hepatocellular carcinoma by disrupting signal transducer and activator of transcription 3 signaling. Hepatology 54: 2137-2148, 2011.

20. Li T, Dong ZR, Guo ZY, Wang CH, Tang ZY, Qu SF, Chen ZT, Li XW and Zhi XT: Aspirin enhances IFN- $\alpha$-induced growth inhibition and apoptosis of hepatocellular carcinoma via JAK1/ STAT1 pathway. Cancer Gene Ther 20: 366-374, 2013.

21. Testoni B, Schinzari V, Guerrieri F, Gerbal-Chaloin S, Blandino $G$ and Levrero M: p53-paralog DNp73 oncogene is repressed by IFN $\alpha / \mathrm{STAT} 2$ through the recruitment of the Ezh2 polycomb group transcriptional repressor. Oncogene 30: 2670-2678, 2011

22. Wan S, Zhao E, Kryczek I, Vatan L, Sadovskaya A, Ludema G, Simeone DM, Zou W and Welling TH: Tumor-associated macrophages produce interleukin 6 and signal via STAT3 to promote expansion of human hepatocellular carcinoma stem cells. Gastroenterology 147: 1393-1404, 2014.

23. Li J, Liu K, Liu Y, Xu Y, Zhang F, Yang H, Liu J, Pan T, Chen J, Wu M, et al: Exosomes mediate the cell-to-cell transmission of IFN- $\alpha$-induced antiviral activity. Nat Immunol 14: 793-803, 2013.

24. Roberts PJ and Der CJ: Targeting the Raf-MEK-ERK mitogenactivated protein kinase cascade for the treatment of cancer. Oncogene 26: 3291-3310, 2007.

25. Franke TF, Kaplan DR and Cantley LC: PI3K: Downstream AKTion blocks apoptosis. Cell 88: 435-437, 1997. 